\title{
Efficacy of Application of Dietary Supplements in Acute Intoxication
}

\author{
Z.B. Yessimsiitova ${ }^{1}$, Saparov Kuandyk ${ }^{1}$, Y. Sinyavskiy ${ }^{2}$, G. Tussupbekova ${ }^{1}$, \\ N. Ablaikhanova ${ }^{1}$, A. Kozhamzarova ${ }^{1}$, Ablaikhanova Nurzat ${ }^{1}$, Ussenova Gauhar $^{3}$, \\ Turlybekov Murat ${ }^{3}$
}

${ }^{1}$ Kazakh National University Named after Al Farabi, Temiryazeva Street, 72, Almaty, Kazakhstan

${ }^{2}$ Academy of Nutrition, Almaty, Kazakhstan

${ }^{3} \mathrm{MTI}$ Medical, Almaty, Kazakhstan

\begin{abstract}
The effectiveness of BAA balsam "Revival plus" as a means of pharmacological correction of neurotoxic and hepatotoxic lesions as consequences of acute ethanol poisoning was evaluated. It has been established that in the conditions of acute severe intoxication ethanol BAA balsam "Revival plus" has pharmacological efficacy in terms of survival keeping temperature and frequency of respiratory movements in poisoned rats with a therapeutic and prophylactic scheme of their use. Value of the work consists in the experimental substantiation of the possibility of using BAA balsam "Revival plus" as a promising means of treatment of acute extremely severe ethanol poisoning.
\end{abstract}

The aim of this paper is to demonstrate the efficacy of application of dietary supplements in acute intoxication, to depict the effectiveness of balsam during the intoxication, and from scientific purpose to prove the significance of this supplement.

The obtained data testify to the effectiveness of the use of bio additives in ethanol poisoning and possible mechanisms for the implementation of the hepatic protective effect of the BAA balsam "Revival Plus" under various life-threatening conditions.

The purpose of this research is evaluation of the experimental effectiveness of BAA balsam "Revival Plus" for ethanol poisoning.

To achieve this goal the following tasks are set:

To reveal the features of the histological structure of the liver of control and experimental rats of the second group with ethanol poisoning;

To study the morphological changes in the liver of rats of the third group with ethanol poisoning against the background of the use of BAA balsam "Revival plus";

To study the peculiarities of the action of the BAA balsam "Revival plus" on the biochemical parameters of the blood of rats.

Questions about the interaction of dietary supplements and alcoholic beverages are regularly being raised by both researchers and ordinary consumers. In most cases talk about the dangers of dietary supplements for a person who often drinks alcoholic beverages is not necessary.

Keywords: Efficacy, application, dietary, supplements, acute, intoxication.

\section{INTRODUCTION}

Biologically active additives are not medicinal products therefore belong to the category of food products which is known as a product do not harm health when interacting with alcoholic beverages. There are dietary supplements which are literally created for interaction with alcohol: they are used to treat and poison the body. Some of them were designed to remove toxic products of alcohol breakdown from the body some to suppress the

Address correspondence to this article at the Kazakh State National University of al-Farabi, Almaty, Kazakhstan; Tel: +77073851878

E-mail: zhanat_2006@mail.ru pleasure that occurs when drinking alcohol to suppress cravings for it.

Currently there is a large number of works describing experimental studies of pathological changes in the body of laboratory animals under the influence of ethanol intoxication usually severe to coma. Medicines including antidotes do not fully solve the problem of providing assistance in acute extremely serious poisoning. The need to improve the pharmacological support of such victims has not lost its relevance since untimely implementation of medical measures leads to the development of early complications long-term consequences and adverse outcomes of poisoning. At the heart of the existing 
arsenal of a pharmacological agent for the treatment of acute intoxication with ethanol the main place is occupied by drugs acting on the leading links in the pathogenesis of acute poisoning [1-3].

The internal environment of the body is represented by tissue fluid, lymph and blood. However the true internal environment of the body is liquid because only it contacts the cells of the body. The blood is in contact with the endothelium of the vessels ensuring their vital functions and only through the tissue fluid interferes with the work of organs and tissues. In general the internal environment of the body is a single system of humoral transport including general circulation, blood $\rightarrow$ interstitial fluid $\rightarrow$ tissue $\rightarrow$ interstitial fluid $\rightarrow$ lymph $\rightarrow$ blood. Blood belongs to the support-trophic group and has a number of features: its constituent parts are formed outside the vascular bed; intercellular substance of blood - liquid; the bulk of the blood is in motion.

Blood and organs in which the formation and destruction of blood cells occurs are united in the blood system. This includes the bone marrow, liver, spleen, lymph nodes [4-7].

The conditions in which we live and with whom we interact can become an external cause provoking different diseases. All exogenous factors can be divided into mechanical, physical, as well as chemical and biological. If we talk about such a factor as malnutrition then it should be recognized that it can cause a variety of disorders of the body provoke protein, carbohydrate, fatty fasting, hypovitaminosis and vitamin deficiency contribute to the development of anemia or even tuberculosis.

The changes in the state of peripheral blood under various endo- and exogenous effects are described in sufficient detail. The most important is the study and selection of funds that affect hematopoiesis and the functional state of the body. For the prevention of listed and other possible diseases activation of anabolic metabolic processes is necessary consisting of intensive elimination of toxic substances from the body and optimization of the supply of the organism with microelements. The accumulation of biometabolites surpluses of medicines and other substances used in the practice of modern sports, alcoholic beverages, stimulants, anabolic steroid hormones and other prohibited preparations, vitamins, etc., is an extremely topical problem that today can be successfully solved only by efferent methods therapy.
Bioactive substances of natural origin of the fifth generation not only actively detoxify, they are still extremely gently and naturally included in the system of antioxidant protection of the body and make up for its functions. They do not interfere with it like most analogous antioxidant drugs, but work directly on the cellular level and in the internal environment of the body which automatically reduces to a minimum the risk of possible negative reactions and complications. For more than ten years the Kazakhstani company MTI MEDICAL has been studying the therapeutic properties of ionic and molecular iodine compounds.

lodine has the property of programmed destruction (apoptosis) of alien RNA viruses and DNA levels, bacteria and parasites. From the moment it hits the infected cell, iodine illuminates and inactivates the foreign microorganism until it completely dies. In this case iodine does not break the cell's own structure. lodine increases the antioxidant status of human serum which prevents the development of infections. lodine in balsam is capable of killing all classes of pathogenic microorganisms.

Most bacteria die within 15 to 30 seconds after contact with iodine. Clinical trials have shown that "Rebirth Plus" balm is not toxic, there is no danger of overdose, undeveloped iodine is naturally excreted through urine, respiration and sweat glands within 27 hours. The drug has pronounced immune stimulatory, hepatic protective, antibacterial, antiviral, detoxifying, antioxidant, anti-parasitic, antiseptic properties.

"REVIVAL plus" balm is a unique complex of lowmolecular compounds of iodine and auxiliary substances. In certain pathological conditions the general therapeutic efficacy of oxidative-free radical detoxification may exceed that of non-oxidant-radical toxins. This postulate is based on the biochemical mechanism of the occurrence of endogenous intoxication when the violation of the integrity of membrane structures due to the intensification of peroxidation triggers a process of limited proteolysis with the subsequent accumulation of products of incomplete metabolism which have a complex toxic effect on organs and systems - liver, kidneys, heart and blood vessels, blood, immunity and etc. [8 -11].

However an evaluation of their effectiveness as a means of prevention and therapy of acute severe alcohol poisoning has not been carried out to date which was the basis for the present work. 


\section{MATERIAL AND METHODS OF RESEARCH}

The experimental study was carried out on the basis of the Laboratory of Evolutionary and Environmental Morphology of the Kazakh National University named after Al-Farabi. In the experiment, 60 white mongrel rats with a body weight of 160-200 grams of the same age were used in laboratory animals and were kept in the same conditions of the vivarium. The use of animals in the experiment was carried out in compliance with the norms and rules regulated by the legislation of the Republic of Kazakhstan and international recommendations of the European Convention for the Protection of Vertebrates used for experiments for scientific or other purposes. During the experiment all the animals were in the same standard vivarium conditions. Decapitation of animals was carried out using narcosis at a strictly fixed time between 9 and 11 o'clock in the morning. The object of histological examination was the main population of liver cells. The whole experiment was carried out for 30 days, all animals were divided into 3 groups: The rats of the control group 1 received aliquots of the physiological solution in the same way as in the experimental rats. The experimental rats of the second group received a $40 \%$ aqueous solution of ethanol in a dose $15 \mathrm{ml} / \mathrm{kg}$ of body weight with daily oral administration for 30 days in two divided doses at 6 hours intervals at the same time of day. The rats of the third group also received a $40 \%$ aqueous solution of ethanol at a dose of $15 \mathrm{ml} / \mathrm{kg}$ of body weight with the use of BAD Balsam "Revival Plus" $0.5 \mathrm{mg} / \mathrm{kg}$ with daily oral administration for 30 days in two divided doses at intervals of 6 hours at the same time of day. On the 30th day of the experiment decapitation was performed under anesthesia. Immediately after decapitation the liver was taken. Decapitation of rats was performed in the morning hours in accordance with existing documents and legislative acts regulating the use of laboratory animals in experimental studies. Morphological examination of liver tissue of experimental animals was performed according to a histological method according to the generally accepted technique of Yu. Volkov. For histological examination pieces of liver tissue from the experimental animals were fixed in a $10 \%$ solution of neutral formalin in $0.1 \mathrm{M}$ phosphate buffer at $\mathrm{pH} 7.2$ 7.4. Dehydration of liver fragments was performed by wiring through a series of isopropanol solutions of the ascending fortress, followed by pouring into paraffin. From the blocks obtained in this manner, sections 3-5 $\mu \mathrm{m}$ in thickness were prepared which were stained with hematoxylin and eosin for the preparation of histological preparations.

\section{DISCUSSION}

All of the above stated the goal of the present study aimed at solving the problems of the development and use of biologically active additives that increase the resistance of the organism to unfavorable factors in everyday life and environment. BAAs have directed physiological and biochemical properties increase the adaptive capacity of the organism in conditions of toxic poisoning. In addition, the use of dietary supplements will increase the resistance of the body and shorten the period of adaptive and re-adaptation disorders in the body. The obtained data can serve as a justification for the use of dietary supplements for therapeutic purposes, as a factor that increases the adaptive capacity of the organism.

An experimental study was performed on 60 experienced male rats, Wistar males, three months old with an initial body weight of 160-200 grams. We detected morph structural changes in the liver, which occurred by the influence of $40 \%$ ethanol and $40 \%$ ethanol + BAA balsam "Revival plus". Special changes in control rats of weakness, malaise, behavioral changes in rats were not detected, the general condition is normal, total weight, pupils and coat were normal, and no physiological abnormalities were observed. In animals of the second group, they experience conditions of toxic poisoning they show excitement and even some aggression. The mass of the organs studied in the control rats was unchanged, and in the restless organs it decreased. Morph functional changes are expressed in rats receiving $40 \%$ ethanol. In the liver of animals after taking $40 \%$ of ethanol during a month, cell degeneration, blurring, and violation of compensatory-adaptive reactions were detected. In animals of the third group, a partial change in the organs was observed in the study of the liver. There is a weakly pronounced interstitial edema, but the beam structure is preserved. Hepatocytes with a clear vacuolated cytoplasm and nuclei. Use of BAA balsam "Revival plus" led to a decrease in the severity of damage to liver tissue. Given that ethanol causes damage to hepatocytes, it can be assumed that under these conditions there will be a deficiency of glucose in the body. A significant decrease in the serum glucose level of animals receiving only ethanol was found, compared to the group of intact animals. At the same time in the group with the application of pharmacological correction of dietary supplements with balsam "Revival plus", the decrease in glucose level was moderate compared to the group of animals that received ethanol. So in the group with the use of BAA 
balsam "Revival plus", the increase in the concentration of ALT was less pronounced compared with the ethanol group. Thus, the conducted studies have made the following conclusions.

\section{CONCLUSION}

The effectiveness of BAA balsam "Revival plus" as a means of pharmacological correction of neurotoxic and hepatotoxic lesions as consequences of acute ethanol poisoning was evaluated. It has been established that in the conditions of acute severe intoxication ethanol BAA balsam "Revival plus" has pharmacological efficacy in terms of survival keeping temperature and frequency of respiratory movements in poisoned rats with a therapeutic and prophylactic scheme of their use.

Theoretical and practical value of the work consists in the experimental substantiation of the possibility of using BAA balsam "Revival plus" as a promising means of treatment of acute extremely severe ethanol poisoning. The obtained data testify to the effectiveness of the use of bio additives in ethanol poisoning and possible mechanisms for the implementation of the hepatic protective effect of the BAA balsam "Revival Plus" under various lifethreatening conditions.

1. Use of BAA balsam "Revival Plus" in the experiment of the third group does not cause in the examined organs of the rats a special change of destructive nature. Minor changes in the histo- structure are completely reversible and have a compensatory-adaptive character.

2. It was found that with the action of $40 \%$ ethanol the picture of the biochemical index of blood changed significantly. BAA balsam "Revival plus" has an ambiguous effect on the biochemical indicators of blood. It should be noted that the biochemical parameters of blood when corrected with BAA balsam "Revival plus" do not exceed the physiological norm.

3. BAA Balsam "Revival Plus" has immune stimulant, hepatic protective properties, antioxidant, detoxifying, antiseptic, responsible for the development of metabolic toxicosis, block the effect of aggressive free radicals and adapt genic properties, increase the body's resistance to infections, toxicity, adverse environmental factors, play an important corrective role.

\section{REFERENCES}

[1] Luzhnikov EA. Poisoning. Small medical encyclopedia. M.: Medicine 1996; 4: 173.

[2] Medical toxicology: National leadership. Ed. E.A. Luzhnikov. Moscow: GEOTAR-Media 2012; p. 928.

[3] Emergency clinical toxicology. Guide for doctors / under. Ed. E.A. Luzhnikov. M.: Medpraktika-M 2007; p. 608.

[4] Markizova NF, Grebenyuk AN, Basharin VA, Bonitenko EYu. Alcohols. SPb.: OOO "Publishing House FOLIO" 2004; p. 112.

[5] Aksauliuk IK, Lapudev AA, Gusarov Al. Emergency medical care for acute poisoning with technical fluids. Mil. Med Journal 1982; 9: 58-59.

[6] Ostapenko UN, Litvinov NN, Rozhkov PG, et al. The concept of reducing the mortality of the population of the Russian Federation in acute poisoning of chemical etiology. In: Problems of the Environment and Natural Resources. M.: VINITI 2009; 7: 3-62.

[7] Necessary VP, Savchuk SA. Alcoholic mortality and toxicity of alcoholic beverages. Partners and competitors. Laboratory 2005; 5: 15-26.

[8] Narcology: national leadership. Under. Ed. N. Ivanets, I.P. Anokhin, M.A. Vinnikov. Moscow: GEOTAR-Media 2008; p. 720.

[9] Luzhnikov EA, Ostapenko YuN, Sukhodolova GN Emergency conditions for acute poisoning. M.: Medpraktika 2001; pp.151-153.

[10] Brothers Elizabeth, Corden Timothy E. Ethanol Toxicity http://misc.medscape.com/pi/iphone/medscapeapp/html/A10 10220-business.html

[11] Matthew J. Ellenhorn. Ellenhorn's Medical Toxicology. Diagnosis and Treatment of Human Poisoning. 2-nd Edition. Williams and Wilkins 1997; pp. 1127-1151. 